\title{
Fabrication and Characterization of Magnetoplasmonic Liposome Carriers
}

\author{
Zahra Hassannejad ${ }^{1,3}$, Mohammad E. Khosroshahi ${ }^{1,2 *}$ and Masoumeh Firouzi ${ }^{4}$ \\ ${ }^{1}$ Laser and Nanobiophotonics Lab, Faculty of Biomedical Engineering, Amirkabir University of Technology, Tehran, Iran \\ ${ }^{2}$ Center of Advance Diffusion and Technology (CADIFT), Department of Mechanical Eng. \& Industry, University of Toronto, Canada \\ ${ }^{3}$ Sina Trauma and Surgery Research Center, Tehran University of Medical Sciences, Iran \\ ${ }^{4}$ Tissue Repair Lab, Institute of Biochemistry and Biophysics, University of Tehran, Iran
}

Received: March 31, 2014; Accepted: March 31, 2014; Published: April 29, 2014

*Corresponding author: Mohammad Khosroshahi, Center of Advance Diffusion and Technology (CADIFT), Department of Mechanical Eng. \& Industry, University of Toronto, MSS $3 G 8$ Canada; Tel:+1- 416-978-1287; E-mail: khosrom@mie.utoronto.ca

\begin{abstract}
Phospholipid liposome encapsulating gold-coated superparamagnetic iron oxide nanostructures is developed. Gold nanoshells were fabricated by a multistep procedure through electroless plating of $\mathrm{Au}$ on the surface of the gold decorated superparamagnetic iron oxide nanoparticles (SPIONs). They were then stabilized using polyvinyl pyrolidone (PVP) with the molecular weight of $25 \mathrm{kDa}$, followed by their encapsulation within hydrophilic core of liposomes, composed of egg yolk phosphatidylcholine (EPC) : cholesterol (CHOL) with a molar ratio of 2:1and an efficiency of $94 \%$. Surface chemistry, hydrodynamic size and electrokinetic potential, morphology and optical properties of magnetoplasmonic liposomes (MPLs) were studied by FT-IR, DLS, TEM and UV-Vis spectroscopy, respectively. FT-IR results showed the presence of amine functional groups on the surface of SPIONs as well as coordination of the nitrogen and oxygen atoms of PVP to the Au atoms at the surface of gold nanoshells. The effect of surface coating on the stability of nanostructures was discussed. The obtained results show that the fabricated hybrid nanostructures are potentially useful for delivery of plasmonic nanoparticles to cells and can be used as exogenous absorber in laser thermal therapy.
\end{abstract}

Keywords: Superparamagnetic iron oxide nanoparticle; Gold nanoshell; PVP; Magnetoplasmonic liposome; Stability

\section{Introduction}

Since the description of unique optical properties of noble metal nanoparticles, which deviate from the bulk materials, by Gustav Mie who solved Maxwell's electrodynamic equations for a homogeneous sphere in 1908 [1], a vast majority of researches have been carried out for the development of noble metal nanoparticles with different structures as well as their applications in biomedicine. Noble metal nanoparticles can be used as a biosensor [2], an exogenous absorber for laserinduced thermal therapy $[3,4]$ or laser soldering [5]. Wavelength of plasmon resonance $\left(\lambda_{\mathrm{SPR}}\right)$, the extinction cross-sections, and the ratio of scattering to absorption efficiency at $\lambda_{\mathrm{SPR}}$ are critical optical properties of noble metal nanoparticles which can be tuned by size, shape, and the dielectric properties of the surrounding medium.
Recently, the influence of assembly of nanoparticles, number of the nanoparticles in the nanocluster as well as the distance of the particles from one another on optical properties of plasmonic nanoparticles has been assessed based on experimental results and theoretical calculations [6-8]. Khlebtsov et al. theoretically evaluated the influence of the number of assembled nanoparticles and the structure of the nanoclusters on the absorption efficiency [6]. Liu et al. theoretically evaluated the dependence of the optical characteristics of gold nanoshells on the inter-particle distance [7]. Also, we recently reported a time dependent post synthesis Doppler shift by gold/SPION nanostructures due to self-assembly [8].

The assembly of plasmonic nanoparticles within the live cells is important in diagnostic and therapeutic applications. Zharov et al. used antibody-conjugated gold nanoparticles to accumulate them within cells [9]. Also, nanoparticles can be embedded within polymeric matrices, microspheres as well as liposomes. Hydrophilic and hydrophobic nanoparticles can be incorporated into the lipid bilayer or the core of the liposome, respectively. Park et al. prepared gold nanoparticles of 3-4 nm loaded into the bilayer of liposomes and evaluated the fluidity changes of bilayer due to nanoparticle loading [10]. Also, Paasonen et al. fabricated multilamellar liposomes with embedded gold nanoparticles of 2-4 $\mathrm{nm}$ [11]. In addition, there are some reports about the deposition of gold nanoparticles onto the surface of liposomes [12-14]. Here, we developed unilamellar liposomal carriers encapsulating PVP-stabilized gold nanoshells. The variation in the $\lambda_{\text {SPR }}$ of gold nanoparticles is too limited. Gold nanoshells are found to have optical cross-sections comparable to and even higher than the nanoparticles. Additionally, it has been reported that the $\lambda_{\mathrm{SPR}}$ can be rapidly increased by either increasing the total nanoshell size or increasing the ratio of the core to shell radius [15].

Encapsulation efficiency is the most important properties of liposomes in drug delivery applications. Here, we applied thin film hydration method to encapsulate stabilized hydrophilic gold nanoshells within the core of liposomes. In addition, the large number of nanoshells per liposome offering the 
advantage of developed hybrid nanostructures for delivery of magnetoplasmonic nanoparticles within a live cell with higher efficiency.

\section{Experimental Section}

\section{Chemical and reagents}

All analytical reagents were used without further purification. Ferric chloride hexahydrate $\left(\mathrm{FeCl}_{3} \cdot 6 \mathrm{H}_{2} \mathrm{O}, 99 \%\right)$, ferrous chloride tetrahydrate $\left(\mathrm{FeCl}_{2} 4 \mathrm{H}_{2} \mathrm{O}, 99 \%\right)$, hydrochloric acid $(\mathrm{HCl}, 37 \%)$, sodium hydroxide, chloroform, formaldehyde solution $_{2} \mathrm{CO}$, $37 \%)$, absolute ethanol and polyvidone25 were purchased from Merck. Gold (iii) chloride trihydrate $\left(\mathrm{HAuCl}_{4} \cdot 3 \mathrm{H}_{2} \mathrm{O}\right.$, $\geq 49 \% \mathrm{Au}$ basis), 3-aminopropyltriethoxysilane (APTES), egg yolk phosphatidylcholine (EPC) and cholesterol (CHOL) were purchased from Sigma. Tetrakis (hydroxymethy1) phosphonim chloride (THPC) was an $80 \%$ aqueous solution from Aldrich. Deionized water (18 M $\Omega$ ) was provided by a Milli-Q system and deoxygenated by vacuum for 1 hour prior to the use.

\section{Synthesis of SPIONs}

SPIONs were synthesized by coprecipitation method [8]. Briefly, iron salts were dissolved in deoxygenated aqueous solution of $0.4 \mathrm{M} \mathrm{HCl}$ where the molar ratio of $\mathrm{Fe}^{3+}: \mathrm{Fe}^{2+}$ was adjusted to 2:1. An instant black precipitate was fabricated by drop wise addition of iron ions solution to $1.5 \mathrm{M} \mathrm{NaOH}$ for 30 min in an inert atmosphere at room temperature. The isolated precipitate was washed with deoxygenated water for 5 times and followed by washing twice using absolute ethanol.

\section{Synthesis of gold coated SPIONs}

Gold coated SPIONs were fabricated by a multistep procedure through electroless plating of $\mathrm{Au}$ onto precursor nanoparticles $[8,16]$. The surface of SPIONs was functionalized with APTES to generate an amine terminated surface [17]. Amine functionalization was performed by adding APTES $(35 \mu \mathrm{l})$ to the suspension of SPIONs in ethanol. $\mathrm{H}_{2} \mathrm{O}(1 \mathrm{ml})$ was introduced into the reaction medium to initiate the hydrolysis. The reaction was allowed to proceed for 7 hours at room temperature and then rinsed by applying an external magnetic field and redispersing with ethanol to remove excess unreacted chemicals. A colloidal gold solution containing $\sim 2 \mathrm{~nm}$ Au particles was prepared using THPC as the reductant according to Duff and Baker [18] method and was refrigerated for 2-3 weeks before use. Precursor nanoparticles were prepared by adding amine terminated SPIONs in ethanol ( $1 \mathrm{ml}$ of $0.0128 \mathrm{M}$ ) into THPC gold solution $(40 \mathrm{ml})$ and $\mathrm{NaCl}(4 \mathrm{ml}$ of $1 \mathrm{M}$ ) and refrigerated for 12 hours. Subsequently, precursor nanoparticles were isolated and redispersed in deoxygenated deionized water with the final concentration of $1.28 \mathrm{mM}$. A plating solution was prepared by mixing $\mathrm{HAuCl}_{4}$ solution (3 ml of $1 \%$ ) with aqueous solution of $\mathrm{K}_{2} \mathrm{CO}_{3}(200 \mathrm{ml}$ of $1.8 \mathrm{mM}$ ) and aged for $24-48 \mathrm{~h}$ in the dark. Subsequently, a continuous gold shell was grown around the SPIONs through reduction of $\mathrm{AuCl}^{4-}$ ions in the plating solution by formaldehyde onto the precursor nanoparticles. Complete shell growth took about 7 min which was monitored by absorbance measurements.

\section{PVP- stabilized gold nanoshells}

PVP solution was prepared by dissolving PVP (27.1 mg / $\mathrm{ml})$ in water and ultrasonication of the solution for $30 \mathrm{~min}$ at room temperature [19]. In order to prepare PVP-coated gold nanoshells, in a typical procedure, obtained nanoshell dispersion in water ( $2 \mathrm{ml}$ ) was centrifuged at $4000 \mathrm{~g}$ for $25 \mathrm{~min}$. Subsequently, supernatant $(1 \mathrm{ml})$ was replaced by PVP solution $(1 \mathrm{ml})$ and the obtained mixture stirred for 24 hours at room temperature. Next, it was centrifuged twice under $4000 \mathrm{~g}$ for $25 \mathrm{~min}$ and washed with Milli-Q water in order to remove free PVP from solution.

\section{Fabrication of liposomal vesicles}

Liposomes were composed of EPC : CHOL at a molar ratio of 2:1. A lipid mixture of chloroform stocks was prepared and dried at $42^{\circ} \mathrm{C}$ under nitrogen stream and further placed in a vacuum overnight. The lipid films were hydrated at a concentration of 20 mM EPL in HEPES buffered saline (HBS, 25 mM HEPES, $140 \mathrm{mM}$ $\mathrm{NaCl}, \mathrm{pH} 7.4$ ) at $65^{\circ} \mathrm{C}$ for $2 \mathrm{~h}$ (vortexed for $30 \mathrm{~s}$ every $5 \mathrm{~min}$ ). After hydration, $15 \mathrm{~min}$ of sonication (Tecna 20, $190 \mathrm{~W}$ ) was applied to break down any larger vesicles. Subsequently, the samples underwent 5 cycles of freeze-thaw including $10 \mathrm{~min}$ at $-196^{\circ} \mathrm{C}$, $10 \mathrm{~min}$ at $65^{\circ} \mathrm{C}$ and $30 \mathrm{~s}$ vortexing between cycles.

\section{Preparation of magnetoplasmonic liposomes}

Liposomal vesicles encapsulating hydrophilic gold nanoshells were prepared according to the procedure described above. In this case, the lipid film was hydrated with the PVP-coated gold nanoshell solution instead of HBS. Unencapsulated nanoparticles were removed by centrifuging at $400 \mathrm{~g}$ for $5 \mathrm{~min}$, after which the supernatant liposomal dispersion was centrifuged at 20000 $\mathrm{g}$ for $30 \mathrm{~min}$ to precipitate the liposomes encapsulating gold nanoshells.

\section{Characterization}

Transmission electron microscopy (TEM) was performed using a CM 200 FEG STEM Philips-M.E.R.C operating at the voltage of $200 \mathrm{kV}$. Liposomes were observed by TEM following negative staining with uranyl acetate. Liposomes were diluted with distilled water and dropped on a PDL-coated copper grid. The excessive sample was removed with filter paper and air-dried for $1 \mathrm{~min}$ at room temperature. Subsequently, uranyl acetate solution $(10 \mu \mathrm{l}$ of $1 \%)$ was dropped onto the grid. After $1 \mathrm{~min}$ the excess staining solution was removed with filter paper and was allowed to dry in the air before introduction into the microscope. Fourier Transform Infrared Spectroscopy (FT-IR) spectra were recorded by TENSOR27 FT-IR spectrometer. The obtained nanoparticles were dried, mixed with $\mathrm{KBr}$ and compressed into a pellet. UVVis spectroscopy of nanoparticle suspensions was taken on a CARY100 UV-Vis spectrophotometer with a $10 \mathrm{~mm}$ optical path length quartz cuvette. The mean particle size and polydispersity of the liposomal vesicles were determined using Dynamic Laser Light Scattering (DLS) method. The scattered light intensity at $90^{\circ}$ was measured by light scattering photometer (Brookhaven instrument). The zeta potential was measured as the particle electrophoretic mobility by means of laser microelectrophoresis in a thermostatted cell at room temperature. Every sample 
measurement was repeated 5 times. Encapsulation efficiency of gold nanoshells within the liposomes was determined by inductively coupled plasma mass spectrometry (ICP-MS). Samples for ICP-MS (VARIAN 735-ES) analysis were frozen, lyophilized, and dissolved in nitric acid hydrochloride, prepared by adding nitric acid $(100 \mu \mathrm{L})$ and hydrochloric acid $(300 \mu \mathrm{L}$ of $37 \%$ ) for $72 \mathrm{~h}$ to dissolve particles. Then, samples were diluted to $2 \mathrm{~mL}$ with $\mathrm{HNO}_{3}(1.6 \mathrm{~mL}$ of $2 \%$ ) and analyzed via ICP-MS against standards [20].

\section{Theory of parameter affecting optical properties of gold nanostructures}

It is known that the optical resonance is a function of size and shape of the nanoparticles. In addition, the surface plasmon peak is greatly influenced by the local dielectric environment [21]. The change of the size of nanoparticles relative to the wavelength of light can produce different order of polarization ranging from the lowest dipolar to higher order multipoles. In the case of particles much smaller than the wavelength of light, the electron oscillation can be considered to be predominantly dipolar in nature. In the dipolar mode, the polarizability of a sphere of volume $\mathrm{V}$ is given by the Clasius-Mossotti relation [22]:

$$
\alpha=3 \varepsilon_{0} V\left(\frac{\varepsilon-\varepsilon_{m}}{\varepsilon+2 \varepsilon_{m}}\right)
$$

where, $\varepsilon_{0}$ is permittivity of vacuum, $\varepsilon_{m}$ is the dielectric constant of the surrounding medium, and $\varepsilon$ is dielectric function of metal.

Equation (1) incorporates the dependence of polarizability on the dielectric function of the metal and polarizability has a strong maximum when $\varepsilon=-2 \varepsilon_{\mathrm{m}}$. Then, an increase of the dielectric constant of the medium results in an increase of negative value of $\varepsilon$ required satisfying the plasmon resonance condition, resulting in a red shift of the plasmon resonance wavelength.

Furthermore, an increase in the medium dielectric constant results in the weakening of the Coulombic restoring force on the displaced electron cloud and hence lowering the plasmon oscillation frequency. Thus, surface plasmon frequency is sensitive to any changes in the refractive index of the local medium around the particle, including adsorption of molecules or change of the solvent. Additionally, plasmon shifts have been observed due to the perturbation of the conduction electron density of the nanoparticles caused by adsorbate-metal interactions [23]. Chemical interaction of the electrons with adsorbates can also results in a broadening of the plasmon resonance band, which is known as chemical interface damping [24].

Moreover, formation of three-dimensional clusters changes the absorption and scattering properties of plasmonic nanoparticles because of the delocalization of the SPR. Correspondingly, the color of a system varies. Cluster formation of plasmon particles, the number of particles in the cluster, as well as the distance between the particles in close approximation changes the intensity and position of $\lambda_{S P R}$ [7-8].

\section{Statistical analysis}

Number of particle size based on TEM micrographs is the average of at least 50 measurements and reported as mean \pm standard deviation. The SPSS 15.0 was used to perform the calculations.

\section{Results and Discussions}

\section{a. FTIR}

FT-IR measurements were carried out to delineate the surface characteristics of nanoparticles. Figure 1 shows the FT-IR spectra of bare SPION (a), amine-functionalized SPION (b), PVP (c) and PVP-covered gold nanoshell (d). The broad band at region of $3550-3200 \mathrm{~cm}^{-1}$ with moderate intensity is assigned to the presence of $-\mathrm{OH}$ groups at the surface of nanoparticles. In Figure 1 (a) the peaks at 444 and $579 \mathrm{~cm}^{-1}$ are assigned to the vibration of Fe-O bond, which are characteristic bands of magnetite. The broad band at $1623 \mathrm{~cm}^{-1}$ and the splitted band at $3414 \mathrm{~cm}^{-1}$ can be attributed to the $\mathrm{N}-\mathrm{H}$ stretching vibration and $\mathrm{NH}_{2}$ bending mode of free $\mathrm{NH}_{2}$ groups, respectively. Also, it can be seen that the characteristic bands of the $\mathrm{Fe}-\mathrm{O}$ bond of amine-functionalized SPIONs shift to higher frequencies of 620 and $477 \mathrm{~cm}^{-1}$ compared with that of bare SPIONs (at 579 and $444 \mathrm{~cm}^{-1}$ ). These shifts have been attributed to the replacement of $-\mathrm{H}$ at the $\mathrm{Fe}-\mathrm{O}-\mathrm{H}$ groups on the surface of SPION by the more electronegative group of -

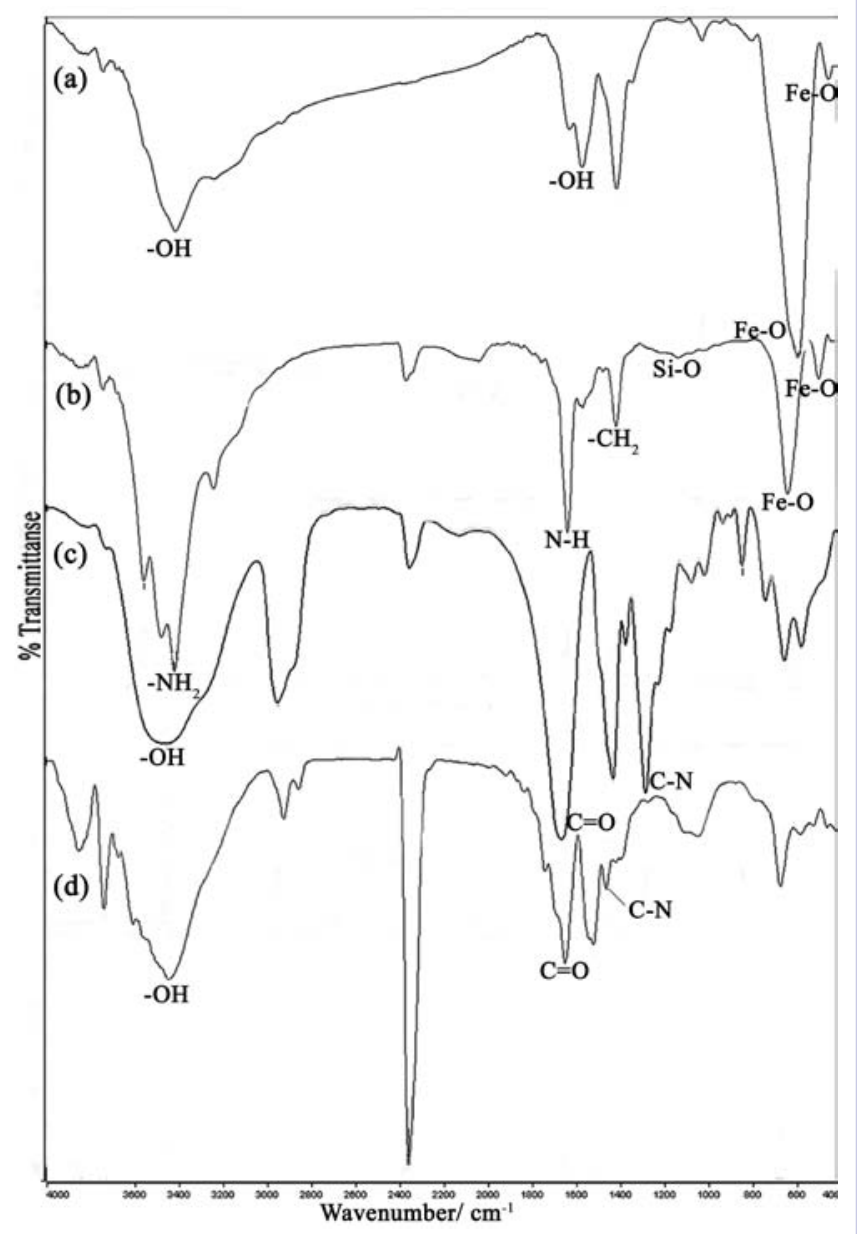

Figure 1: FT-IR spectra of bare SPION (a), amine-functionalized SPION (b), PVP (c) and PVP-covered gold-coated SPION (d). 
$\mathrm{Si}\left(\mathrm{O}-\mathrm{J}_{2}\right.$ - which leads to the enhancement of bond force constant for Fe-O bonds [25].

The FT-IR spectrum of PVP (Figure 1c) mainly consists of two bands at 1282 and $1664 \mathrm{~cm}^{-1}$ corresponding to the vibration of C-N and carbonyl group in pyrrolidone ring [26]. Similar bands are also seen in the FT-IR spectrum of PVP-coated gold nanoshells. However, comparing with the spectrum of PVP, the resonance peak of $\mathrm{C}-\mathrm{N}$, at $1282 \mathrm{~cm}^{-1}$, was shifted to $1475 \mathrm{~cm}^{-1}$ and the band of $\mathrm{C}=0$, at $1664 \mathrm{~cm}^{-1}$, red shifted to $1647 \mathrm{~cm}^{-1}$. The change of the spectrum indicates that the absorption of PVP on the gold shell is not on the basis of electrostatic attraction but $\mathrm{Au}$ atoms on the surface of nanoparticles would coordinate with $\mathrm{N}$ and $\mathrm{O}$ atoms of PVP which is consistent with previously reported results [27].

\section{b. DLS}

According to the Derjarguin-Landau-Verwey-Overbeck theory (DLVO) theory, the stability of colloidal suspension results from the equilibrium between attractive (i.e. van der Waals) and repulsive (i.e. electrostatic) forces. For magnetic suspensions, magnetic dipolar forces between two particles must be added in order to promote the attraction force between the particles. Thus, electrical double layer is not enough to prevail against to coagulation in many conditions such as changing the $\mathrm{pH}$ value and ionic strength or in physiological medium. Coating of nanoparticles by polymers develops steric repulsion force which depends on molecular weight of the polymer and its density. Controlling the strength of these forces is a key parameter to elaborate nanoparticle suspensions with good stability.

In aqueous solutions, the $\mathrm{Fe}$ atoms coordinate with water molecules, which dissociate readily to leave a hydroxylated surface. The hydroxyl groups on the surface of iron oxide are amphoteric and may react with acids or bases. Dependent on the $\mathrm{pH}$ of the solution, the relative abundance of protonated or deprotonatonated hydroxyl groups governs the electrokinetic potential of iron oxide particles in the absence of absorbing ions other than $\mathrm{H}^{+}$. The corresponding surface reactions may expressed as:

$$
\begin{aligned}
& \equiv \mathrm{FeOH}+\mathrm{H}^{+} \leftrightarrow \equiv \mathrm{FeOH}_{2}^{+} \\
& \equiv \mathrm{FeOH} \leftrightarrow \mathrm{FeO}^{-}+\mathrm{H}^{+}
\end{aligned}
$$

In our case, an electrokinetic potential of $-29.7 \pm 0.68 \mathrm{mV}$ at neutral $\mathrm{pH}$ was obtained for bare SPIONs which is consistent with previously reported values [28]. Subsequently, surface hydroxyl groups exchanged by amine groups through silanization reaction using APTES. The zeta potential of amine-functionalized SPIONs increased to $+16.6 \pm 1.62 \mathrm{mV}$. The $\mathrm{p} K_{\mathrm{a}}$ value of amine groups is about 10 and at neutral $\mathrm{pH}$ they are protonated. Therefore, the increase of the zeta potential to positive value indicates the successful grafting of APTES on the surface of SPIONs. The zeta potential value of gold colloids with an average size of $\sim 2 \mathrm{~nm}$ formed using THPC reducing agent was $-40.91 \pm 1.14 \mathrm{mV}$. Therefore, by mixing of gold colloids with amine-functionalized SPIONs in a mixture of water and ethanol with a $\mathrm{pH}$ value of 9 , the negatively charged gold colloids readily attached to the SPIONs led to the decrease of the zeta potential value of amine-functionalized SPION to -19.17 \pm 0.27 . The zeta potential of gold nanoshells and the surface potential of PVP-coated gold nanoshells were measured -21.63 \pm 0.9 and $-22.77 \pm 0.73$, respectively.

The measured properties of synthesized nanoparticles are summarized in Table 1 . The effect of surface coating on the stability of SPIONs is observable from DLS data. The volumeweighted hydrodynamic size measured by DLS for SPIONs, amine-functionalized SPIONs, gold nanoshells and PVP-coated nanoshells was 201.4, 95.4, 57.7 and $102.5 \mathrm{~nm}$, respectively. The reduced hydrodynamic size of amine-functionalized SPIONs shows more stability of nanoparticle suspension after silanization reaction. This fact confirms that, apart from the electrostatic repulsion due to a zeta potential of $+16.6 \pm 1.62$, steric repulsion arising from grafting of the APTES to the nanoparticles increased the stability of amine-functionalized SPIONs. Coating of the amine-functionalized SPIONs with a continuous thin layer of gold reduced the hydrodynamic size of gold nanoshells to 57.7 $\mathrm{nm}$. At temperatures below the blocking SPIONs have a zero magnetic moment. However, it has shown that residual forces, of magnetic dipolar origin, acting between SPIONs lead them to clump [29]. The reduced size of gold-coated SPIONs could be due to the reduction of this residual forces. After PVP coating of gold nanoshells the hydrodynamic size increased to $102.5 \mathrm{~nm}$ which is because the immobilization of long chain molecules of PVP at the surface of the nanoparticles. This result agrees well with the previously reported thickness in the case of PVP adsorbed on palladium nanoparticles [30]. Fluctuation in the measured hydrodynamic size of synthesized nanoparticles is illustrated in Figure 2.

\section{c. TEM}

The bright field TEM micrographs of particles at different steps of nanoshell fabrication are presented in Figure 3. Based on TEM micrographs, the average diameter of SPIONs and gold nanoshells was $9.5 \pm 1.4$ and $15.8 \pm 3.5 \mathrm{~nm}$, respectively. The larger size of SPIONs and gold nanoshells measured by DLS compared with TEM results indicates the slight agglomeration (Figure 3A and $3 \mathrm{C}$, respectively).

The TEM micrograph of MPLs is shown in Figure 4. Encapsulation of a large number of nanoparticles into the hydrophilic inner site of liposome indicates high encapsulation

Table 1: Properties of synthesized nanoparticles in different steps of

\begin{tabular}{|c|c|c|c|}
\hline Samples & $\begin{array}{l}\text { TEM size }(\mathrm{nm}) \\
\pm \mathrm{SE}\end{array}$ & $\begin{array}{l}\text { DLS volume- } \\
\text { weighted } \\
\text { size (nm) } \\
\text { (PDI) }\end{array}$ & $\begin{array}{l}\text { Electropho- } \\
\text { retic potenti- } \\
\text { al }(\mathrm{mV}) \pm \mathrm{SE}\end{array}$ \\
\hline SPION & $9.5 \pm 1.4$ & $201.4(0.224)$ & $-29.79 \pm 0.68$ \\
\hline SPION-NH2 & --- & $95.4(0.283)$ & $+16.60 \pm 1.62$ \\
\hline Gold colloid & $2.34 \pm 0.62$ & -- & $-40.91 \pm 1.14$ \\
\hline Precursor NP & --- & --- & $-19.17 \pm 0.27$ \\
\hline SPION/gold nanoshell & $15.8 \pm 3.5$ & $57.7(0.25)$ & $-21.63 \pm 0.9$ \\
\hline PVP-coated nanoshell & --- & $102.5(0.288)$ & $-22.77 \pm 0.73$ \\
\hline $\begin{array}{l}\text { Nanoshell-encapsulated } \\
\text { liposome }\end{array}$ & $179.73 \pm 69.93$ & $290.5(0.311)$ & $-40.91 \pm 1.14$ \\
\hline
\end{tabular}
fabrication. 

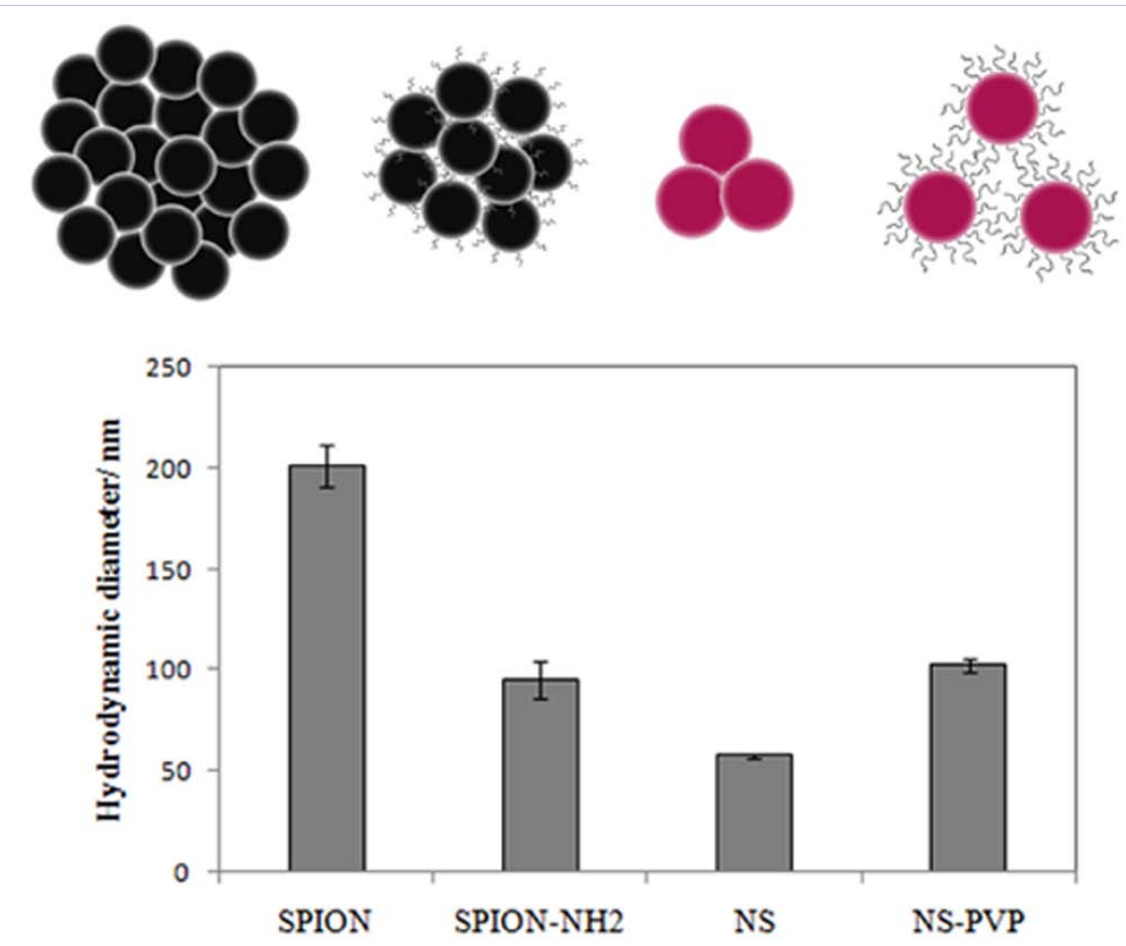

Figure 2: Schematic representation of the fluctuation in the measured hydrodynamic size of SPIONs, amine-functionalized SPIONs (SPION-NH2), gold nanoshells (NS) and PVP-coated gold nanoshells (NS-PVP) is shown in the upper part of the figure and the histogram of hydrodynamic diameter of mentioned nanoparticles is shown in the lower part of the figure.

efficiency of the prepared magnetoplasmonic liposmes. Also, the clear margin of magnetoplasmonic liposmes in TEM micrographs indicates that a collection of PVP-coated gold nanoshells surrounded by a lipid bilayer. An average diameter of 179.73 $\pm 69.93 \mathrm{~nm}$ was obtained for magnetoplasmonic liposmes. The thickness of the lipid bilayer membrane surrounding the collection of nanoshells was $6.5 \pm 1.6 \mathrm{~nm}$ measured by NIH ImageJ software (http://rsb.info.nih.gov/ij/). Similarly, a value of 7-8 $\mathrm{nm}$ was also reported for lipid bilayer membrane [31]. These data suggest that the formed vehicles have unilamellar liposomal structure.

\section{d. Encapsulation efficiency}

Encapsulation efficiency of gold MPLs was calculated using ICP-MS results through the formula $\left(\mathrm{W}_{1} / \mathrm{W}\right) \times 100 \%$, where $\mathrm{W}$ is initial concentration of $\mathrm{Fe}(\mathrm{mg} / \mathrm{ml})$ in hydration solution and $W_{1}$ is encapsulated concentration of $\mathrm{Fe}(\mathrm{mg} / \mathrm{ml})$ in liposomes. An encapsulation efficiency of $94 \%$ was obtained. In order to evaluate the effect of PVP coating on encapsulation efficiency, we also fabricated magnetoplasmonic liposmes using as-synthesized gold nanoshells (without PVP coating). An encapsulation efficiency of $24 \%$ was obtained. These results indicate that stability of hydration suspension has a great effect on encapsulation efficiency of liposomes.

In some reports, the encapsulation efficacy of prepared nanoparticle-loaded liposomes has not been reported $[11,13]$ and this makes the comparison difficult. However, the encapsulation efficiency of our prepared nanoparticle-loaded liposomes is more than those reported by Sabaté et al. [32] for similar nanoparticle concentration used to prepare magnetic nanoparticle-loaded liposomes.

Furthermore, as mentioned above, TEM micrograph of magnetoplasmonic liposmes show that the obtained liposome has a unilamellar structure. In previous reports, it has been shown that sonication can break down the large liposomes. Sonication can also produce unilamellar liposomes [33]. Therefore, our hypothesis is that stabilization of gold nanoshells through PVP coating and application of sonication during the fabrication procedure resulted in the formation of unilamellar liposomes with a high gold nanoshell encapsulation efficiency.

\section{e. Optical properties of fabricated MPLs}

As shown in Figure 5, the surface plasmon resonance wavelength of synthesized gold nanoshells was $540 \mathrm{~nm}$ which red shifted to $554 \mathrm{~nm}$ after coating with PVP. As mentioned in the theory, this red shift can be due to the increased dielectric constant of the medium after coating with PVP. Also, encapsulation of PVP-coated nanoshells within liposomal structure caused to damping and broadening of absorption band. This broad band can be attributed to the size distribution of prepared MPLs and contribution from different number of gold nanoshells collected within liposomes.

Furthermore, Visual observation of MPL solution (Figure 5) confirms the high encapsulation efficiency. The difference between colors of aqueous dispersion of PVP-coated gold nanoshells compared with gold nanoshell-encapsulated 

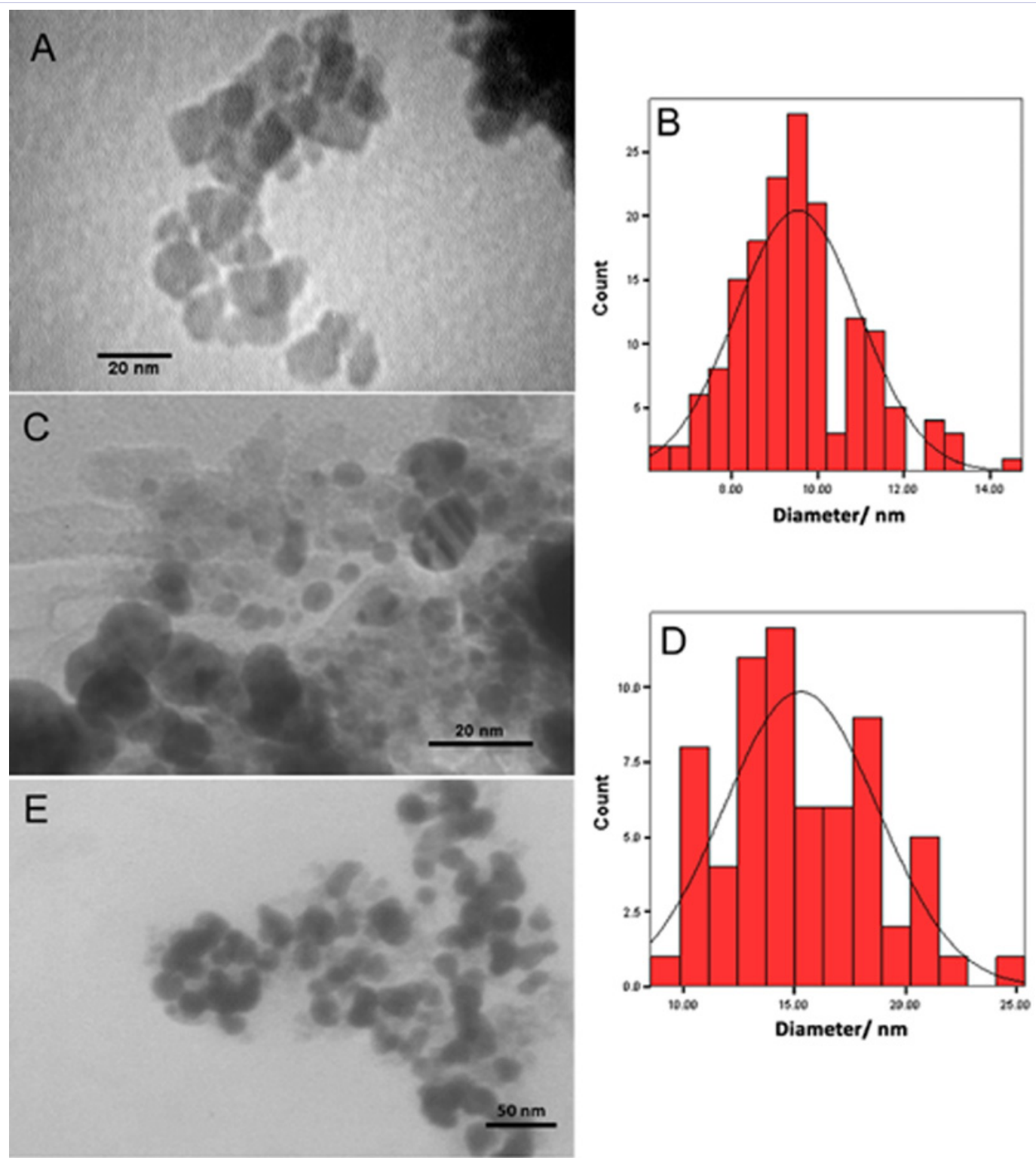

Figure 3: TEM images showing (A) SPIONs of diameter $9.5 \pm 1.4 \mathrm{~nm}$ and (B) its corresponding size distribution, (C) gold nanoshells of diameter 15.3 $\pm 3.5 \mathrm{~nm}$ and (D) its corresponding size distribution and (E) PVP-coated gold nanoshells.

liposomes can be due to the encapsulation of a collection of gold nanoshells within the inner part of the liposomes. Physical nature of these optical effects is related to strong electromagnetic interaction between plasmon nanoparticles due to assembly. In addition, the change of the medium refractive index caused by phospholipids encapsulating nanoshells can result in the shift of the $\lambda_{S P R^{*}}$ Although, the complexes of gold nanoparticles with liposomes are known [34], to the best of our knowledge, the present study is the first report on the gold nanoshell-loaded liposomes.

Delivery of gold nanostructures to desirable cells using targeting moiety or carries (e.g. liposomes or polyelectrolyte hollow capsules) can be used to accumulate nanoparticles directly within live cells. It has been observed that the closed packing of the nanoparticles resulted in non-linear or synergistically amplified photothermal effects, strongly enhancing their diagnostic and therapeutic ability $[9,35,36]$. Recently, the influence of nanoparticle aggregation on the efficiency of electromagnetic absorption has been studied in order to lower the threshold fluence level for biomedical applications such as laser-induced photothermal therapy. Optical amplification photothermal therapy with plasmonic nanoclusters has been reported by [6]. In order to evaluate the maximal absorption of single particle and cluster structures, they introduced the parameter of absorption amplification which defines the enhancement of cluster absorption cross section $C_{a b s}$ in comparison with the simple sum of absorption cross section for isolated nanoparticles $N C_{a b s}$. They found that when the number of cluster nanoparticles $N$ is small, the electromagnetic coupling results in an increase in 


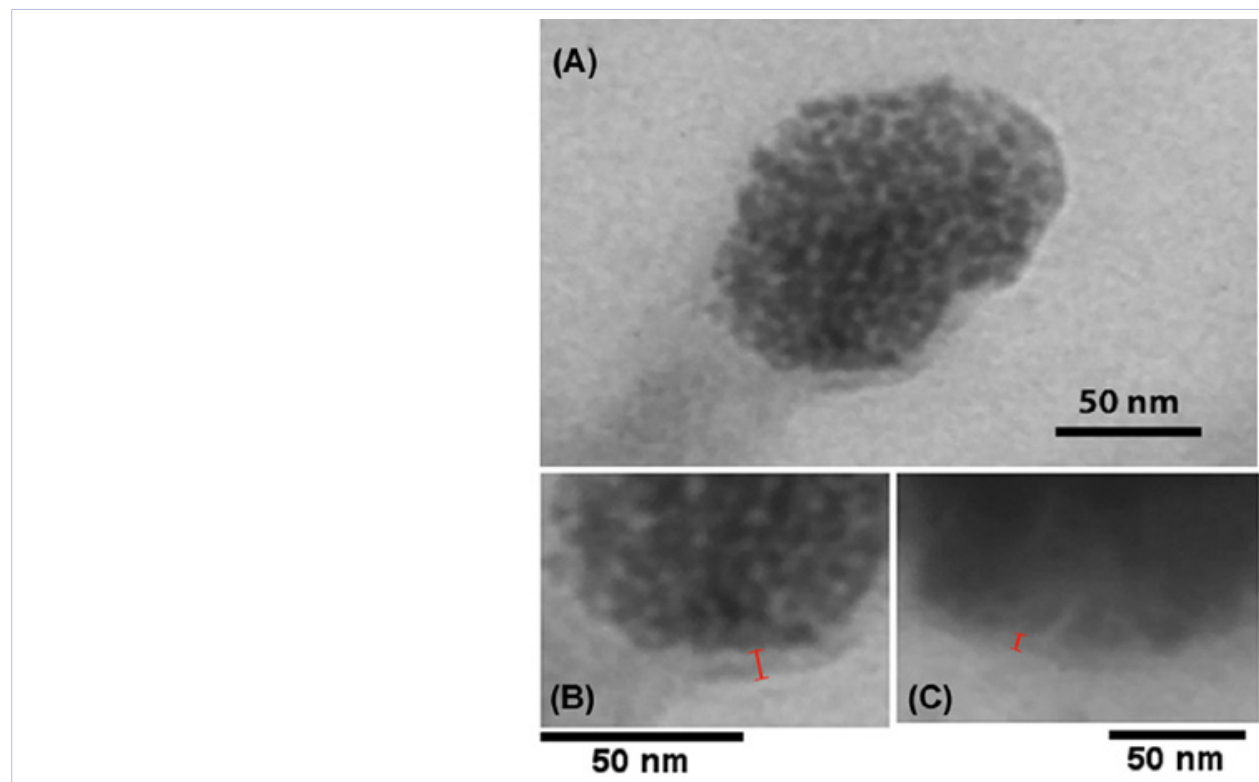

Figure 4: (A) TEM image of gold nanoshell-encapsulated liposome showing the phospholipid bilayer (visualized by negative staining using $1 \%$ uranylacetate) surrounding a collection of PVP-coated gold nanoshells. In (B) and (C) the bilayer thickness is indicated by red lines.
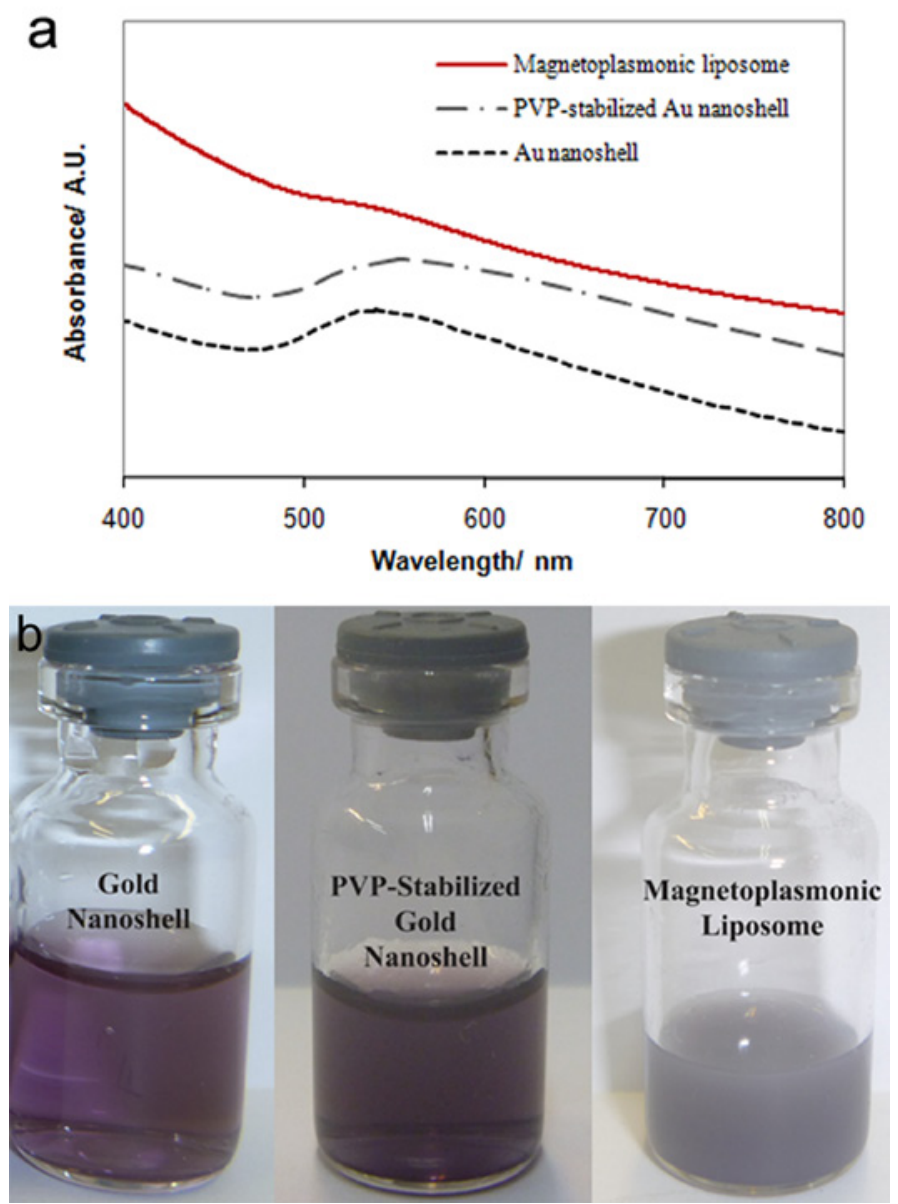

Figure 5: (A) Absorption measurements of gold nanoshell, PVP-coated gold nanoshell and nanoshell-encapsulated liposome. Optical images of aqueous dispersion of gold nanoshells, PVP-coated gold nanoshells and gold nanoshell-encapsulated liposomes are shown in (B). 
the absorption efficiency and reaches a saturated value at $N>20$. Then, the obtained hybrid structure in the present study could be a good candidate for laser-induced thermal therapy application.

\section{Conclusion}

Fabrication of a new class of nanoparticle-encapsulated liposomes was provided. Gold nanoshells have recently attracted attention for biomedical applications such as laser-induced thermal therapy. Stability of large number of gold nanoshells and their transport into the cells are the critical factors which have to be optimized for biomedical applications. Fluctuation of hydrodynamic size of synthesized nanoparticles measured by DLS showed the effect of coating on the stability of gold nanoshells. PVP-stabilized gold nanoshells were encapsulated in the hydrophilic core of phospholipid liposomes to achieve 94\% encapsulation efficiency. Also, TEM micrographs of MPLs revealed the collection of nanoparticles within a liposome which makes the prepared unilamellar liposome a good vehicle to transport a collection of nanoparticles within the cells. In addition, evaluation of optical properties of nanoparticles during the development of hybrid structure show the influence of the refractive index of the surrounding medium as well as the cluster formation on the position and the intensity of surface plasmon resonance wavelength.

\section{References}

1. Craig FB, Donald RH (1983) Absorption and Scattering of Light by Small Particles. Inc : John Wiley \& Sons, New York, USA.

2. Khlebtsov BN, Khlebtsov NG (2007) Biosensing potential of silica/ gold nanoshells: Sensitivity of plasmon resonance to the local dielectric environment. J Quant Spectrosc Radiat Transfer 106(1-3): 154-169.

3. C S R, Kumar J, V R, M V, Abraham A (2012) Laser immunotherapy with gold nanorods causes selective killing of tumour cells. Pharmacol Res 65(2): 261-269.

4. Melancon MP, Lu W, Zhong M, Zhou M, Liang G, et al. (2011) Targeted multifunctional gold-based nanoshells for magnetic resonance-guided laser ablation of head and neck cancer. Biomaterials 32(30): 7600-7608.

5. Khosroshahi M, Nourbakhsh MS (2011) In vitro skin wound soldering using $\mathrm{SiO}_{2} / \mathrm{Au}$ nanoshells and a diode laser. Medical Laser Application 26(1): 35-42.

6. Khlebtsov B, Zharov V, Melnikov A, Tuchin V, Khlebtsov N (2006) Optical amplification of photothermal therapy with gold nanoparticles and nanoclusters. Nanotechnology 17(20): 5167-5179.

7. Liu C, Mi CC, Li BQ (2008) Energy Absorption of Gold Nanoshells in Hyperthermia Therapy. IEEE Trans Nanobioscience 7 (3): 206-214.

8. Zahra H, Mohammad EK (2013) Synthesis and Evaluation of Time Dependent Optical properties of Plasmonic-Magnetic Nanoparticles. Opt Mater 35(3): 644-651.

9. Zharov VP, Mercer KE, Galitovskaya EN, Smeltzer MS (2006) Photothermal Nanotherapeutics and Nanodiagnostics for Selective Killing of Bacteria Targeted with Gold Nanoparticles. Biophys J 90(2): 619-627.

10. Park SH, Oh SG, Mun JY, Han SS (2006) Loading of gold nanoparticles inside the DPPC bilayers of liposome and their effects on membrane fluidities. Colloids Surf B Biointerfaces 48(2): 112-118.

11. Paasonen L, Sipilä T, Subrizi A, Laurinmäki P, Butcher SJ et al. (2010)
Gold-embedded photosensitive liposomes for drug delivery: Triggering mechanism and intracellular release. J Controlled Release 147(1): 136-143.

12. Tapan KS, Alexander SU, Srujan KD, Michael F, Margaret RH, et al. (2009) Controlling loading and optical properties of gold nanoparticles on liposome membranes. Colloids and Surfaces 342(1-3): 92-96.

13. Paasonen L, Laaksonen T, Johans C, Yliperttula M, Kontturi K, et al. (2007) Gold nanoparticles enable selective light-induced contents release from liposomes. J Controlled Release 122(1): 86-93.

14. Leung SJ, Kachur XM, Bobnick MC, Romanowski M (2011) WavelengthSelective Light-Induced Release from Plasmon Resonant Liposomes. Adv Funct Mater 21(6): 1113-1121.

15. Jain PK1, Lee KS, El-Sayed IH, El-Sayed MA (2006) Calculated Absorption and Scattering Properties of Gold Nanoparticles of Different Size, Shape, and Composition: Applications in Biological Imaging and Biomedicine. J Phys Chem B 110 (14): 7238-7248.

16. Brinson BE, Lassiter JB, Levin CS, Bardhan R, Mirin N, et al. (2008) Nanoshells Made Easy: Improving Au Layer Growth on Nanoparticle Surfaces. Langmuir 24(24): 14166-14171.

17. Mohammad EK, Lida G (2012) Synthesis and functionalization of $\mathrm{SiO}_{2}$ coated $\mathrm{Fe}_{3} \mathrm{O}_{4}$ nanoparticles with amine groups based on self-assembly. Materials Science and Engineering C 32(5): 1043-1049.

18. Daniel GD, Alfons B, Peter PE (1993) A New Hydrosol of Gold Clusters. 1. Formation and Particle Size Variation. Langmuir 9(9): 2301-2309.

19. Graf C, Dembski S, Hofmann A, Rühl E (2006) A General Method for the Controlled Embedding of Nanoparticles in Silica Colloids. Langmuir 22(13): 5604-5610.

20. von Maltzahn G, Park JH, Agrawal A, Bandaru NK, Das SK, et al. (2009) Computationally guided photothermal tumor therapy using longcirculating gold nanorod antennas. Cancer Res 69(9): 3892-3900.

21. Nanomaterials Chemistry: Recent Developments and New Directions (2007) Rao CNR, Müller A, Cheetham AK (eds) Wiley-VCH Verlag $\mathrm{GmbH} \& \mathrm{Co} . \mathrm{KGaA}$.

22. K Lance Kelly, Eduardo Coronado, Lin Lin Zhao , and George C. Schatz (2002) The Optical Properties of Metal Nanoparticles: The Influence of Size, Shape, and Dielectric Environment. J Phys Chem B 107(3): 668-677.

23. Paul M, Jorge PJ, Michael G, Luis ML, Carlos P (2006) Drastic Surface Plasmon Mode Shifts in Gold Nanorods Due to Electron Charging. Plasmonics 1(1): 61-66

24. Hövel H, Fritz S, Hilger A, Kreibig U, Vollmer M (1993) Width of cluster plasmon resonances: Bulk dielectric functions and chemical interface damping. Phys Rev B 48(24): 18178-18188.

25. Ming M, Zhang Y, Wei Y, Hao-ying S, Hai-qian Z, et al. (2003) Preparation and characterization of magnetite nanoparticles coated by amino silane. Colloids and Surfaces A 212(2-3): 219-226.

26. Sen M, Avci EN (2005) Radiation synthesis of poly(N-vinyl-2pyrrolidone)-kappa- carrageenan hydrogels and their use in wound dressing applications. I. Preliminary laboratory tests. J Biomed Mater Res A 74(2): 187-196.

27. HongLing L, Peng H, Weng XZ, JunHua W (2010) Synthesis of monosized core-shell $\mathrm{Fe} 3 \mathrm{O} 4 / \mathrm{Au}$ multifunctional nanoparticles by PVP-assisted nanoemulsion process. Colloid Surf A 356(1-3): 21-27.

28. Zhong-Xi S, Fen-Wei S, Willis F, Per-Olof S (1998) Surface Characteristics of Magnetite in Aqueous Suspension. J Colloid Interface Sci 197(1): 151159.

29. Dobson JF, Gray EM (2009) Residual attractive force between 
superparamagnetic nanoparticles. 1-21.

30. Hidefumi H, Noboru Y (2001) Protecting polymers in suspension of metal nanoparticles. Polymers of Advanced Technologies 12(11-12): 724-733.

31. Alice F, Andrea A, Anna M, Giorgio P, Anna MF (2011) SPION@ liposomes hybrid nanoarchitectures with high density SPION association. Soft Matter 7(13): 6239-6247.

32. Sabate' R, Barnadas-Rodri'guez R, Callejas-Ferna'ndez J, HidalgoAlvarez R, Estelrich J (2008) Preparation and characterization of extruded magnetoliposomes. Inter J Pharmaceutics 347(1-2): 156-162.

33. Kim SH, Haimovich-Caspi L, Omer L, Talmon Y, Franses EI (2007) Effect of sonication and freezing-thawing on the aggregate size and dynamic surface tension of aqueous DPPC dispersions. J Colloid Interface Sci 311(1): 217-227.

34. Kojima C, Hirano Y, Yuba E, Harada A, Kono K (2008) Preparation and characterization of complexes of liposomes with gold nanoparticles. Colloid Surf Biointerfaces 66(2): 246-252.

35. Zharov VP, Galitovskaya EN, Johnson C, Kelly T (2005) Synergistic enhancement of selective nanophotothermolysis with gold nanoclusters: Potential for cancer therapy. Lasers Surg Med 37(3): 219-226.

36. Zharov VP, Kim J-W, Curiel DT, Everts M (2005) Self-assembling nanoclusters in living systems: application for integrated photothermal nanodiagnostics and nanotherapy. Nanomedicine 1(4): 326-345. 